\title{
Effective treatment with a tetrandrine/chloroquine combination for chloroquine-resistant falciparum malaria in Aotus monkeys
}

\author{
Zuguang Ye ${ }^{1}$, Knox Van Dyke $2^{2^{*}}$ and Richard N Rossan ${ }^{3,4}$
}

\begin{abstract}
Background: In vitro evidence indicates that tetrandrine (TT) can potentiate the action of chloroquine 40-fold against choloquine-resistant Plasmodium falciparum. The key question emanating from that study is "would tetrandine and chloroquine be highly effective in a live Aotus monkey model with chloroquine-resistant parasites". This study was designed to closely mimic the pharmacological/anti-malarial activity in man.

Methods: The Vietnam Smith/RE strain of P. falciparum, which is chloroquine-resistant was used in this study. Previous experimental procedures were followed. Panamanian owl monkeys (Aotus) were inoculated with $5 \times 10^{6}$ erythrocytes parasitized with the CQ-resistant strain of $P$. falciparum. Oral drug treatment was with CQ (20 mg/kg) and/or tetrandrine at $15 \mathrm{mg} / \mathrm{Kg}, 30 \mathrm{mg} / \mathrm{Kg}$ or $60 \mathrm{mg} / \mathrm{Kg}$ or $25 \mathrm{mg} / \mathrm{Kg}$ depending on experimental conditions.

Results and Discussion: Parasitaemia was cleared rapidly with CQ and $\Pi T$ while CQ treatment alone was ineffective. Recrudescence of malaria occurred after seven days post-infection. However, four animals were treated orally with $\Pi$ and $C Q$ parasites were cleared. It is likely that monkeys were cured via a combination of both drug and host immune responses. A single Aotus monkey infected with $P$. falciparum and untreated with drugs, died.

No side effects were observed with these drug treatments.

Conclusions: This combination of chloroquine and tetrandrine forms the basis of a new attack on chloroquineresistant malaria - one based upon inhibition of the basis of chloroquine resistance, the multiple drug resistance pump. Previous studies demonstrated that the parasite MDR pump was found on parasite membranes using $3 \mathrm{H}$ azidopine photoaffinity labelling.

Since MDR-based choloroquine resistance is induced by chloroquine, the basis of the action of tetrandrine is the following: 1) tetrandrine inhibits the MDR pump by stimulating MDR ATPase which limits the energy of the pump by depletion of parasite ATP, 2) tetrandrine blocks the genetic factor which controls the induction of the pump. Therefore, it appears that the parasite cannot outsmart these mechanisms and produce a new mode of resistance. Only time will tell if this is correct.
\end{abstract}

Keywords: Tetrandrine, Chloroquine, Aotus monkeys, Chloroquine-resistant falciparum malaria, Synergism

\footnotetext{
* Correspondence: kvandyke@ hsc.wvu.edu

${ }^{2}$ Department of Biochemistry, Robert C Byrd Health Sciences Center of West

Virginia University, 1 Med Center Drive, Morgantown, WV 26506, USA

Full list of author information is available at the end of the article
} 


\section{Background}

Chloroquine has been, and probably still is, the most used anti-malarial drug throughout the world. The emergence and spread of chloroquine (CQ)-resistant falciparum malaria has evoked a search for new drugs effective against this drug-resistant parasite. However, the malarial parasite rapidly develops resistance to new antimalarial drugs; for example, mefloquine and, more recently, artemisinin [1]. In 1987, Martin and co-workers explored a different method to attack the drug resistant parasite with the use of racemic verapamil combined with CQ. This combination reversed the resistance of the parasite in vitro [2], similar to its action in drug resistant cancer. However, at the extensive doses which reversed chloroquine resistance, verapamil exhibits cardiac toxicity in humans, which makes it impractical clinically.

Since then, much work has been focused on reversal of the resistance to CQ by using various compounds, such as calcium channel blockers [3] and their isomers [4-6], antidepressive drugs [7-9], antihistaminics [10-17]. However, most of the above combinations, which reversed the resistance in vitro, have not been successful in vivo either using the Aotus monkey [18] or doing clinical trials [19]. It has been previously reported that tetrandrine, a bisbenzyl isoquinoline, possesses a unique anti-malarial activity, especially selective against CQresistant Plasmodium falciparum [20], and that a combination of tetrandrine with CQ displayed a strong synergism against both CQ-sensitive and CQ-resistant $P$. falciparum in vitro [21]. The data obtained in vitro indicated that this TT/CQ combination was able to increase anti-malarial potency of chloroquine, such that a standard dose of CQ (1.5 g/patient) presumably might actually cure chloroquine-resistant falciparum malaria in humans.

Initially, an in vitro system was used to find active anti-malarial drugs or drug combinations. The situation in vivo is much more complex since biological activity is greatly affected by absorption (if given orally), distribution, metabolism, and excretion of the drug. Therefore, the data obtained in vitro cannot be directly extrapolated in vivo. Results with the tetrandrine-CQ combination in vitro must be tested in an in vivo system.

This study uses Aotus monkeys infected with a CQresistant strain of $P$. falciparum to confirm the effectiveness of the combination against CQ-resistant parasites in vivo. The aim of this study was to provide a pharmacodynamic basis for a possible human clinical trial with this combination.

\section{Methods}

The Vietnam Smith/RE strain of P. falciparum, which is CQ-resistant, was used in this study. Experimental procedures described previously were followed $[18,22]$.
Briefly, Panamanian owl monkeys (Aotus lemurinus lemurinus)(Figure 1) were inoculated intravenously with $5 \times 10^{6}$ erythrocytes parasitized with this CQ-resistant strain of $P$. falciparum. Five days after the inoculation, parasitaemia in the monkeys was about $5 \times 10^{3} / \mathrm{mm}^{3}$, when the treatment with the combination initiated. In conducting the research described in this report, the investigators adhered to "Guide for the Care and Use of Laboratory Animals, prepared by the Committee on Care and Use of Laboratory Animals of the Institute of Laboratory Animals Resources of the Institute of Animal Resources, National Council ( DHEW publication No. (NIH) 78-23 Revised 1978).

A water-solution of both CQ diphosphate and tetrandrine dihydrochloride was prepared respectively at appropriate concentrations. Just before treatment, the two drug solutions were mixed and given orally by gastric intubation in a volume of $7.0 \mathrm{ml}$, followed by $7.0 \mathrm{ml}$ water for rinse.

Treatment regimens in the experiments were divided into two categories. One-week therapy: After infection with the CQ-resistant parasite, the monkey was treated consecutively for seven days with CQ $(20 \mathrm{mg} / \mathrm{kg})$ combination with different doses of tetrandrine ranging from 15 to $60 \mathrm{mg} / \mathrm{kg}$. If recrudescence occurred, the dose of

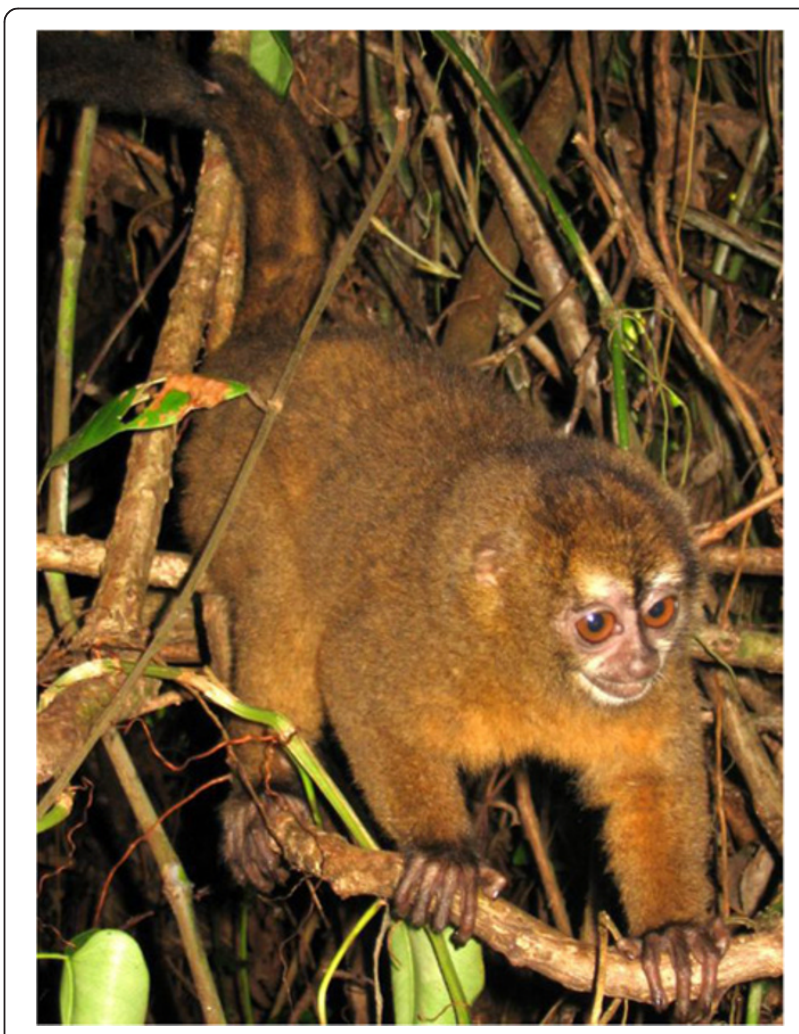

Figure 1 The picture below is an Aotus monkey similar to the animals used in this study. The original source was wikimedia commons and is used with permission. 
tetrandrine in the combination was increased two times the initial dose for another one-week therapy. Two-week therapy: Instead of an increased dose of tetrandrine, the duration of treatment was changed from one week to two weeks.

A control, infected monkey was not given drug. In addition, five infected monkeys were administered CQ orally alone for seven consecutive days.

Blood smears were made daily during the experiment to monitor parasitaemia. After clearance of parasitaemia, a three-month follow-up was done to ascertain cure of infections.

\section{Results}

Two experiments with Aotus monkeys treated with the combination of tetrandrine and CQ were conducted. In the first experiment, $15 \mathrm{mg} / \mathrm{kg}$ tetrandrine and $20 \mathrm{mg} / \mathrm{kg}$ CQ were given orally to three monkeys infected with CQ-resistant P. falciparum for seven consecutive days. In the second experiment, two monkeys were treated with the combination of 30 or $60 \mathrm{mg} / \mathrm{kg}$ tetrandrine and $20 \mathrm{mg} / \mathrm{kg}$ CQ for seven consecutive days. The results are summarized in Table 1.

The CQ-resistant $P$. falciparum parasites were cleared in a mean of 9.2 days, ranging from 7 to 16 days after initial treatment with the drug combination (Table 1). Infections recrudesced in all the treated monkeys within one month after clearance of the parasite. In the first experiment, monkeys No. 025 and 016 were treated for another week with a higher dose of tetrandrine $(30 \mathrm{mg} / \mathrm{kg})$ combined with the CQ $(20 \mathrm{mg} / \mathrm{kg})$ after recrudescence occurred. One of the two monkeys was cured following the second treatment.

Following the recrudescence in the second experiment, re-treatment of monkeys No. 705 and 706 for one week with a higher dose of tetrandrine $(60 \mathrm{mg} / \mathrm{kg})$ did not cure the infections (recrudescence occurred again), although parasitaemia was rapidly cleared. A two-week therapy with a lower dose of tetrandrine $(25 \mathrm{mg} / \mathrm{kg})$ combined with the same dose of CQ (20 mg/kg) was used to treat monkeys No. 705, 706 and 725. No recrudescence occurred during the post-treatment examination period.

The infected monkey without any drug treatment died within two weeks. In monkeys treated with CQ alone, the parasite response to CQ alone was none or a slight suppression. The parasitaemia in three of five monkeys treated with CQ alone increased continuously during the treatment with $C Q$, while the parasitaemia in the remaining two monkeys was suppressed slightly.

\section{Discussion}

The results obtained in Aotus monkeys infected with CQ-resistant P. falciparum showed that the tetrandrine combination with $\mathrm{CQ}$ can rapidly clear parasitaemia, while the parasite is not affected or only slightly affected

Table 1 Effect of the combination of tetrandrine and chloroquine against infected chloroquine-resistant Plasmodium falciparum malaria in Aotus monkeys

\begin{tabular}{|c|c|c|c|c|c|c|c|}
\hline Expt. no. & Monkey no. & $\operatorname{Dose}^{a}(M g / k g)$ & $\begin{array}{l}\text { Parasites } \\
\text { cleared }\end{array}$ & $\begin{array}{c}\text { Days from initial } \\
\text { dose to parasite } \\
\text { clearance }\end{array}$ & $\begin{array}{c}\text { Days from final } \\
\text { dose to recrudescence }\end{array}$ & $\begin{array}{l}\text { Re-treating-post } \\
\text { recrudescence } \\
(\mathrm{mg} / \mathrm{kg})\end{array}$ & Cured \\
\hline \multirow[t]{6}{*}{1} & 025 & $15(\mathrm{tt})$ & + & & & $30(\mathrm{tt})^{\mathrm{d}}$ & $\overline{(++)}$ \\
\hline & & $20(\mathrm{cq})$ & & 16 & 23 & $20(\mathrm{cq})$ & \\
\hline & 055 & $15(\mathrm{tt})$ & + & & & $30(t t)^{d}$ & $\overline{(++)}$ \\
\hline & & 20(cq) & & 8 & 33 & $20(\mathrm{cq})$ & \\
\hline & 016 & $15(\mathrm{tt})$ & + & & & $30(\mathrm{tt})^{\mathrm{d}}$ & $\frac{(++)}{(+)}$ \\
\hline & & $20(\mathrm{cq})$ & & 8 & 13 & $20(\mathrm{cq})$ & \\
\hline \multirow[t]{5}{*}{2} & 705 & $30(\mathrm{tt})$ & + & & & $60(\mathrm{tt})^{\mathrm{d}}$ & $\overline{(++)}$ \\
\hline & & 20(cq) & & 7 & 9 & $20(\mathrm{cq})$ & \\
\hline & 706 & $60(\mathrm{tt})$ & + & & & $120(\mathrm{tt})^{d}$ & $\overline{(++)}$ \\
\hline & & 20(cq) & & 7 & 14 & $20(c q)$ & \\
\hline & $698^{b}$ & - & - & & & & \\
\hline \multirow[t]{5}{*}{$\mathrm{CQ}^{c}$} & 520 & $20(\mathrm{cq})$ & - & & & & \\
\hline & 432 & 20(cq) & - & & & & \\
\hline & 349 & $20(\mathrm{cq})$ & - & & & & \\
\hline & 544 & $20(\mathrm{cq})$ & - & & & & \\
\hline & 631 & $20(c q)$ & - & & & & \\
\hline
\end{tabular}

$\mathrm{a}$ tt and cq represent tetrandrine and chloroquine respectively.

b This monkey as a non-treated control died within 2 weeks after inoculation with the parasite.

c Monkeys were treated with $C Q$ alone.

d Monkeys were cured after two week combinational cq and tt treatment $=(++)$. 
by CQ alone (see Table 1 ). This is similar to a report that the Vietnam Smith/RE strain of $P$. falciparum is very resistant to $\mathrm{CQ}$, because the drug exerted limited effects on parasitaemia in Aotus monkeys that received $20 \mathrm{mg} /$ $\mathrm{kg} /$ day of CQ for several days [18]. Tetrandrine in combination with CQ may either increase total anti-malarial activity or inhibit the resistance of the parasite to CQ or both, so that a dose of CQ $(20 \mathrm{mg} / \mathrm{kg})$ in combination with tetrandrine exerts a remarkably potent anti-malarial effect against the CQ-resistant P. falciparum strain. Later work in cancer indicates that tetrandrine inhibits the transcription factor nuclear factor kappa b. This transcription factor is now known to control the stimulation of multiple drug resistance (MDR) mechanism. This cancer MDR mechanism is quite similar to the MDR pumping system that causes the exit pumping of chloroquine in drug resistant malarial parasites. Therefore, it should not be surprising that tetrandrine could inhibit either or both the production of the pump or the pumping action of the chloroquine- resistance pump (MDR). In fact, these events both happen in MDR drug resistant cancer cells. The tetrandrine/CQ combination creates a major synergism that was observed in the in vitro studies with chloroquine resistance in human falciparum malaria [21].

Recrudescence occurred after the one-week treatment with the drug combination. Following recrudescence in the first experiment, re-treatment of the monkeys with a larger dose of tetrandrine combined with chloroquine did clear the resistant malaria and cured infection in one monkey without the second treatment. In the second experiment, re-treatment with a higher dose of tetrandrine in the combination did not eliminate all the parasites in the monkeys, although the dose of tetrandrine in the combination was increased up to $120 \mathrm{mg} / \mathrm{kg}$. Apparently, the increased dose of tetrandrine does not overcome the basis of recrudescence. Therefore, the length of therapeutic regimen was changed from one week to two weeks. No recrudescence occurred after the twoweek treatment. It seemed that increasing the period of drug exposure to the parasite might be an effective method to overcome recrudescence. However, the likely possibility that host defense was partly responsible for the cure of these monkeys cannot be ruled out, because the monkeys that were treated with the two-week therapy are the same animals that had already received-the one-week treatment twice before; i.e., these monkeys had gone through a process of malarial infection: 7 days treatment - clearance of parasitaemia - recrudescence 7 days retreatment - clearance of parasitaemia - recrudescence. In addition, it has been previously recognized there is some self-cure or spontaneous recovery that occurs naturally in these monkeys infected with $P$. falciparum. Maybe the cure of falciparum infection in these monkeys was elicited either by an effect of the two-week treatment, or partially by a spontaneous recovery, or more likely a combination of the drugs and this host defense. Therefore, it cannot be stated with certainty that the drugs were entirely responsible for the cure. Although the cause of recrudescence is not clear, the following possibilities were considered. Either the initial duration of exposure of the parasite to the drug combination is too short to kill all the parasites, or the concentration of tetrandrine is not enough for a long enough time at the site of action so that parasites that are hiding can survive, or both.

Tetrandrine is a bisbenzyl isoquinoline alkaloid, isolated from Stephania tetrandra, which has been used in traditional Chinese medicine either as an anti-rheumatic or analgesic agent. Tetrandrine is an approved drug in China, which has been used to treat hypertension and silicosis for centuries since it exerts known pharmacological activities on the cardiovascular system and produces anti-inflammatory activity [23-26]. Tetrandrine was found to exert little toxicity in clinical practice [23]. It can be given orally to humans at the dose of $200 \mathrm{mg}$ or $300 \mathrm{mg} /$ day for three months when used for treatment of silicosis [23]. Hence, tetrandrine is a relatively safe drug. Although no toxicological studies per se were performed on these monkeys, using the tetrandrine/CQ combination in this experiment; the monkeys appeared to easily tolerate orally a high dose of $120 \mathrm{mg} / \mathrm{kg}$ of tetrandrine in the combination with CQ at a dose of 20 $\mathrm{mg} / \mathrm{kg}$. One reason for other drug combinations failing when used at high doses in in vivo experiments, including clinical trials, was linked to their inherent toxicity. It seems that tetrandrine has relatively low toxicity. Therefore, it may be practical and helpful to conduct a clinical trial on the combination of tetrandrine and CQ on a small scale after further verifying the combinational safety of the tetrandrine with $\mathrm{CQ}$.

\section{Conclusions}

Based on data obtained in Aotus monkeys infected with CQ-resistant parasites, it can be concluded that the tetrandrine-CQ combination is effective against chloroquine-resistant falciparum malaria in Aotus monkeys. This combination can clear the parasitaemia rapidly, although some recrudescence occurred after treatment. In addition, a longer period of treatment; for example, a two-week's application, might solve the recrudescence problem caused by the shorter treatment (one-week therapy). Artemisinin and its derivatives also displayed recrudescence in clinical trials as well as in animal experiments. By prolonging duration of the treatment with artemisinin or its derivatives, the recrudescence problem was essentially resolved. A further study of the effectiveness of a two-week treatment with the CQ/tetrandrine 
drug combination using a total of five Aotus monkeys to confirm the curative effectiveness of the two-week therapy is warranted.

There was not sufficient funds to continue these studies. Although statistical analysis was not accomplished, the cure of all five drug-treated animals after the 7-14 day combinational treatment leads one to an obvious conclusion that the combination of chloroquine and tetrandrine with an intact host immune system is an effective treatment of Aotus-infected falciparum malaria if treated for the correct amount of time. Both tetrandrine and chloroquine have been used in man as single entities for years. By combining the drugs to treat chloroquine resistant falciparum malaria in man would likely be an effective combination in man without major toxicity from either drug alone. Tetrandrine is metabolized in man and animals with great difficulty because it is a weak substrate for liver p-450 oxidation system. However, only a study in man with chloroquine-resistant, falciparum malaria could reveal the necessary data to confirm this research in Aotus monkeys. Since MDRbased choloroquine resistance is induced by chloroquine [27], the basis of the action of tetrandrine is the following: 1) tetrandrine inhibits the MDR pump by stimulating MDR ATPase which limits the energy of the pump by depletion of parasite ATP, 2) tetrandrine blocks the genetic factor which controls the induction of the pump. Therefore, it appears that the parasite cannot outsmart these mechanisms and produce a new mode of resistance. Only time will tell if this is correct.

\section{Competing interest}

None of the three authors have a known conflict of interest with any material from this manuscript.

\section{Authors' contribution}

RNR performed the experimental/pharmacological work with Aotus monkeys at The Gorgas Memorial Laboratory in Panama. He was involved in both conception and design of this study. ZY was the first scientist to discover the effects of tetrandrine on the actual mechanism of chloroquine resistance. He developed the treatment schedule for this study and was involved with both conception and design of this study. The first effective in vitro screening system for anti-malarial drugs was developed in the laboratory of KVD for the United States Army during 1966-1970 during the Viet Nam war. KVD supplied the tetrandrine and chloroquine for the study and helped in study conception and experimental design. Both ZY and KVD wrote this manuscript. All authors read and approved the final manuscript.

\section{Author details}

${ }^{1}$ Department of Pharmacology, Institute of Chinese Materia Medica, Academy of Traditional Chinese Medicine, Beijing 100700, China. ${ }^{2}$ Department of Biochemistry, Robert C Byrd Health Sciences Center of West Virginia University, 1 Med Center Drive, Morgantown, WV 26506, USA. ${ }^{3}$ Gorgas Memorial Laboratory, Panama, Panama. ${ }^{4}$ Present address: Las Vegas, Nevada 89147, USA.

Received: 31 July 2012 Accepted: 21 March 2013 Published: 2 April 2013

\section{References}

1. Palmer KJ, Holliday SM, Brogden RN: Mefloquine. Drugs 1993, 45:430-475.
2. Martin S, Oduola A, Milhous W: Reversal of chloroquine resistance in Plasmodium falciparum by verapamil. Science 1987, 235:89-91.

3. Tanabe K, Kato M, Izumo A, Hagiwara A, Doi S: Plasmodium chabaudi: in vivo effects of $\mathrm{CA}^{2+}$ antagonists on chloroquine-resistant and chloroquine-sensitive parasites. Exp Parasitol 1990, 70:419-426.

4. Ye Z, Van Dyke K: Reversal of chloroquine resistance in falciparum malaria: independent of calcium channels. Biochem Biophys Res Comm 1988, 155:476-481.

5. Basco LK, Le Bras J: Plasmodium falciparum: In vitro drug interaction between chloroquine and enantiomers of amlodipine. Exp Parasitol 1991, 72:262-270.

6. Deloron P, Basco LK, Dubois B, Gaudin C, Clavier F, Le Bras J, Verdier F: In vitro and in vivo potentiation of chloroquine against malaria parasites by an enantiomer of amlodipine. Antimicrob Agents Chemother 1991, 35:1338-1342.

7. Bitonti AJ, Sjoerdsma A, McCann PP, Kyle DE, Oduola AMJ, Rossan RN, Milhous WK, Davidson DE Jr: Reversal of chloroquine resistance in malaria parasite Plasmodium falciparum by desipramine. Science 1988, 242:1301-1303.

8. Salama A, Facer CA: Desipramine reversal of chloroquine resistance in wild isolates of Plasmodium falciparum. Lancet 1990, 335:164-165.

9. Basco LK, Le Bras J: Reversal of chloroquine resistance with desipramine in isolates of Plasmodium falciparum from Central and West Africa. Trans R Soc Trop Med Hyg 1990, 84:479-481.

10. Peters W, Ekong R, Robinson BL, Warhurst DC, Pan XQ: Antihistaminic drugs that reverse chloroquine resistance in Plasmodium falciparum. Lancet 1989, ii:334-335.

11. Bitonti AJ, McCann PP: Desipramine and cyproheptadine for reversal of chloroquine resistance in Plasmodium falciparum. Lancet 1989, ii:1281-1283.

12. Basco LK, Le Bras J: Desipramine and cyproheptadine for reversal of chloroquine resistance in Plasmodium falciparum. Lancet 1989, ii:1282-1283.

13. Basco LK, Le Bras J: Reversal of chloroquine resistance with cyproheptadine in 'wild's strains of Plasmodium falciparum. Trans $R$ Soc Trop Med Hyg 1991, 85:204-205.

14. Peters W, Ekong R, Robinson BL, Warhurst DC, Pan XQ: The chemotherapy of rodent malaria. XLV. Reversal of chloroquine resistance in rodent and human Plasmodium by antihistaminic agents. Ann Trop Med Parasitol 1990, 84:541-551.

15. Basco LK, Ringwald P, Le Bras J: Chloroquine-potentiating action of antihistaminics in Plasmodium falciparum in vitro. Ann Trop Med Parasitol 1991, 85:223-228.

16. Walter RD, Seth M, Bhaduri AP: Reversal of chloroquine resistance in Plasmodium falciparum by CDR 87/209 and analogues. Trop Med Parasitol 1993, 44:5-8.

17. Chandra S, Ohnishi ST, Dhawan BN: Reversal of chloroquine resistance in murine malaria parasites by prostaglandin derivatives. Am J Trop Med Hyg 1993, 48:645-651.

18. Kyle DE, Milhous WK, Rossan RN: Reversal of Plasmodium falciparum resistance of chloroquine in Panamanian Aotus monkeys. Am J Trop Med Hyg 1993, 48:126-133.

19. Warsame M, Wernsdorfer WH, Bjorkman A: Lack of effect of desipramine on the response to chloroquine of patients with chloroquine-resistant falciparum malaria. Trans R Soc Trop Med Hyg 1992, 86:235-236.

20. Ye Z, Van Dyke K: Selective antimalarial activity of tetrandrine against chloroquine resistant Plasmodium falciparum. Biochem Biophys Res Comm 1989, 159:242-248.

21. Ye Z, Van Dyke K, Castranova V: The potentiating action of tetrandrine in combination with chloroquine or qinghaosu against chloroquinesensitive and resistant falciparum malaria. Biochem Biophys Res Comm 1989, 165:758-765.

22. Rossan RN, Harper JS III, Davidson DE Jr, Escajadillo A, Christensen HA: Comparison of Plasmodium falciparum infections in Panamanian and Colombian owl monkeys. Am J Trop Med Hyg 1985, 34:1037-1047.

23. Anonymous: Clinical observation on the treatment of simple silicosis with tetrandrine. Chin J Ind Hyg Occup Dis 1983, 1:136-139.

24. King VF, Garcia ML, Himmel D, Reuben JP, Lam YT, Pan J, Han G, Kaczoroqski GJ: Interaction of tetrandrine with slowly inactivating calcium channels. J Biol Chem 1988, 263:2238-2244.

25. Seow WK, Ferrante A, Li S, Thong YH: Suppression of human monocyte interleukin-1 production by the plant alkaloid tetrandrine. Clin Exp Immunol 1988, 75:47-51. 
26. Seow WK, Ferrante A, Summors A, Thong YH: Comparative effects of tetrandrine and berbamine on production of the inflammatory cytokines interleukin-1 and tumor necrosis factor. Life Science 1992, 50:53-58.

27. Swyers JP: Overcoming multiple drug resistance in malaria. Res Resour Rep 1990, 14:1-4.

doi:10.1186/1475-2875-12-117

Cite this article as: Ye et al:: Effective treatment with a tetrandrine/

chloroquine combination for chloroquine-resistant falciparum malaria in

Aotus monkeys. Malaria Journal 2013 12:117.

\section{Submit your next manuscript to BioMed Central and take full advantage of:}

- Convenient online submission

- Thorough peer review

- No space constraints or color figure charges

- Immediate publication on acceptance

- Inclusion in PubMed, CAS, Scopus and Google Scholar

- Research which is freely available for redistribution 